\title{
Frequency of Depressive Disorders among Women after Miscarriage
}

\author{
Aafia Malik11, Aneel Shafi², Irum Umair³, Saqib Maraj Aslam Bajwa4 ${ }^{4}$ Aysha Butt ${ }^{1}$ and Fatima Bukharie ${ }^{5}$ \\ ${ }^{1}$ Department of Psychiatry, Jinnah Hospital, Lahore, Pakistan \\ 2 Department of Psychiatry, Avicenna Medical College, Lahore, Pakistan \\ ${ }^{3}$ Department of Psychiatry, Sharif Medical and Dental Hospital, Lahore, Pakistan \\ ${ }^{4}$ Department of Psychiatry, Gujranwala Medical College, Gujranwala, Pakistan \\ ${ }^{5}$ Department of Psychiatry, Sheikh Zayed Hospital, Lahore, Pakistan
}

\begin{abstract}
Objective: To ascertain the frequency of depressive disorder in women after miscarriage.

Study Design: Cross-sectional study.

Place and Duration of Study: The Outdoor Department of Gynaecology and Obstetrics, Services Hospital, Lahore, from January to June 2017.

Methodology: A total of 150 women from Outdoor Department of Gynecology and Obstetrics, Services Hospital, Lahore, fulfilling the inclusion criteria, were registered. Clinical assessment and the diagnosis for depressive disorder was performed by psychiatrist by putting criteria given in International Classification of Disease: $10^{\text {th }}$ Revision (ICD-10). ICD 10 criteria require at least 2 symptoms out of three core symptoms (depressed mood, loss of interest, and reduced energy) along with at least 2 other symptoms, to be diagnosed as depression (ICD 10 criteria is given in annexure 2 of our article as well). The whole data was analyzed on SPSS-10 and p-value was measured. Chi-square was the test of significance. Results: The 150 cases, $62 \%(n=93)$ were in age of $15-30$ years while $38 \%(n=57)$ were in age of $31-40$ years, with mean of $29.17 \pm 5.94$ years, frequency of depressive disorder among women after miscarriage was recorded in $15.33(n=23)$. Stratification for frequency of depressive disorder among women after miscarriage with relation to family history shows that 10 out of 57 cases had family history, while 13 out of 93 cases had no family history of depression $(p=0.55)$.

Conclusion: The frequency of depressive disorder was not very high among women after miscarriage; however, by taking appropriate interventions like guiding the family members, including friends, to provide emotional support to the women after miscarriage, it may further reduce.
\end{abstract}

Key Words: Miscarriage, Depressive disorder, Frequency.

How to cite this article: Malik A, Shafi A, Umair I, Bajwa SMA, Butt A, Bukharie F. Frequency of depressive disorders among women after miscarriage. J Coll Physicians Surg Pak 2020; 30(2):192-196.

\section{INTRODUCTION}

Depression is very common worldwide. It is a syndrome of symptoms such as persistent low mood, lack of pleasure, and absence of interest in daily life activities. In clinical depression, patient can experience guilt, worthlessness, hopelessness, and loss of self esteem. Depression causes great distress and loss of function for the sufferer. Its prevalence during pregnancy was estimated at $7 \%$ in Australia and Taiwan, $8 \%$ in Chile, 9\% in Canada, ranging in the United States from 7 to $13 \%$, and from 7 to $8 \%$ in Europe. ${ }^{1}$ The premature expulsion of an embryo till 23 weeks of pregnancy and weighing till 500 grams is miscarriage. ${ }^{2}$ It is concluded that $17 \%$ of pregnancies in United States end in miscarriage. 3 According to an estimate, one million women in the USA and 70,000 - 90,000 women in the

Correspondence to: Dr. Aafia Malik, Department of Psychiatry, Jinnah Hospital, Lahore, Pakistan

E-mail: aafiamalik@hotmail.com

Received: January 18, 2019; Revised: June 25, 2019;

Accepted: August 08, 2019
UK each year are being affected by pregnancy loss associated with miscarriage. Approximately, ranges in $14 \%$ to $20 \%$ of clinically presented pregnancies end in miscarriage. 4

As miscarriage is so common, the mental health impact of miscarriage is often overlooked; and the affected mental health can have serious consequences for the woman and family members. 5 The impact of miscarriage can be intense behavioral disturbance for many affected females. Mostly, symptoms of depressive disorder, anxiety, grief (pathological), and PTSD are experienced. Factors include age, previous miscarriages, or social support may influence the level of emotional distress. ${ }^{6}$ Mothers in low-income countries have high rates of pregnancy loss and often experience more than one loss. ${ }^{3}$

The outcome and risk regarding mental disorders, especially depression after abortion, should be assessed as soon as possible because major depressive disorder, anxiety disorder including post-traumatic stress disorder, and substance-use disorder were the outcome studied to the highest degree in the research papers published 
in 1995 and 2011.7 It was evident that women who described receiving low support from their social networks were at increased risk of developing postpartum depression compared with women who received high level of support. ${ }^{8}$

A longitudinal cohort study identified the risk factors of depressive disorder after miscarriage: depressive disorder was found to be $10 \%$, anxiety disorder was $1.2 \%$, and two subjects had post-traumatic stress disorder $(0.6 \%)$ and obsessive compulsive disorder $(0.6 \%)$. Risk factors after miscarriage causing depression were history of infertility, younger age, and previous case of depression. ${ }^{9}$

As there was no related local study, the rational of this study is to highlight the frequency of depressive disorder in women after miscarriages in Pakistan; and result will be shared with gynecologists, obstetricians and medical physicians to emphasize on detection of depression after miscarriages in women and referral to mental health care / psychiatrist so that there will be early diagnosis and treatment, which will help in creating awareness and timely intervention. The rational is that the knowledge of frequency may permit health-care workers to initiate proper proceeding like guiding the family unit to provide emotional assistance to the women after miscarriage. Thus this will enhance their psychological well-being or reduce the unfavourable effects of miscarriage on their family relationship and will improve the overall quality of life.

The objective of the study was to ascertain the frequency of depressive disorder in women after miscarriage.

\section{METHODOLOGY}

Its a cross-sectional study, which was channelized in obstetrics and gynaecology of Services Hospital for six months from January to June 2017. The measured sample size is 150 women with $5 \%$ error margin, and $95 \%$ confidence level taking expected percentage of depression among women after miscarriage i.e.10\%. ${ }^{9}$ The sampling technique was non-probability, consecutive sampling. Inclusion criteria were women having the premature expulsion of an embryo till 23 weeks of pregnancy and weight less than 500 grams, of reproductive age (15-49 years) having miscarriage for the first age, whether primi-gravida or multi-gravida, within the last three months. The subjects were excluded, if women with miscarriage had psychiatric morbidity proir to their abortion. Women diagnosed with any gynecological problem, e.g. fibroids, endometriosis or poly-cystic ovaries or having multiple miscarriages, history of chronic liver disease, diabetes mellitus (BSF $>126 \mathrm{mg} / \mathrm{dl}$ ), hypertension (>160/90 mmHg), metabolic disorder, hepatitis $B$ and $C$ and tuberculosis assessed by laboratory investigations (LFTs, HbA1c, serum hepatitis $\mathrm{B}$ and $\mathrm{C}$ and sputum etc.), were excluded. Women who opted for abortion by their own will and those who were taking any psychiatric medication prior to the study were also excluded.

A total of 150 women from Outpatient Department of Obstetrics of the Hospital, who fulfilled the inclusion criteria were registered. The nature of study was explained to each individual and Informed-consent was taken. Confidentiality of bio-data and individual findings were maintained. Demographic data, i.e. age, gender, area of residence, education, marital status, obstetric and gynecological history, monthly income and family size etc., were collected. The diagnosis of depressive disorder was given by applying criteria described in International Classification of Disease-10th revision (ICD-10).

All the collected data were analyzed in SPSS version 10 and the sample size calculation was based upon WHO software/formula for sample size determination. The calculated sample size was 150 women with $5 \%$ margin of error, and $95 \%$ confidence level taking expected percentage of depression among women after miscarriage i.e. $10 \% .9$ The sampling technique was nonprobability, consecutive sampling. Qualitative data like gender and depression were presented as frequency and percentage. Quantifiable data like age was presented as mean \pm standard deviation. Data was stratified to address effect modifiers like age, educational status, and family history etc. Post-stratification, Chi-square was applied to analyze stratified groups. $P$-value $\leq 0.05$ was taken as significant.

\section{RESULTS}

On hundred and fifty cases, fulfilling the inclusion criteria were registered to ascertain the frequency of depressive disorder among women after miscarriage. Age use $62 \%$ $(n=93)$ were in $15-30$ years of age, while $38 \%(n=57)$

Table I: Stratification for frequency of depressive disorder among women after miscarriage in regard to age, educational status and family history $(n=150)$.

\begin{tabular}{|c|c|c|c|}
\hline & \multicolumn{2}{|c|}{$\begin{array}{c}\text { Depressive } \\
\text { disorder }\end{array}$} & \multirow[t]{2}{*}{ p-value } \\
\hline & Yes & No & \\
\hline \multicolumn{4}{|c|}{ Age of pregnant women in years } \\
\hline $15-30$ & 17 & 76 & 0.20 \\
\hline $31-49$ & 6 & 51 & \\
\hline \multicolumn{4}{|c|}{ Educational status of pregnant women in the study } \\
\hline \multicolumn{4}{|c|}{ Illiterate } \\
\hline Yes & 6 & 17 & 0.004 \\
\hline No & 74 & 53 & \\
\hline \multicolumn{4}{|l|}{ Middle } \\
\hline Yes & 11 & 12 & 0.17 \\
\hline No & 42 & 85 & \\
\hline \multicolumn{4}{|c|}{ Matric or higher } \\
\hline Yes & 6 & 17 & 0.01 \\
\hline No & 11 & 116 & \\
\hline \multicolumn{4}{|c|}{ Family history of depression } \\
\hline Yes & 10 & 47 & 0.55 \\
\hline No & 13 & 80 & \\
\hline
\end{tabular}


were ranges in 31-40 years of age, mean $+S D$ was calculated as $29.17 \pm 5.94$ years. Frequency of depressive disorder among women after miscarriage was recorded in $15.33(n=23)$, while $84.67 \%(n=127)$ had no findings of depressive psychiatric morbidity.

Categorization for frequency of depressive disorder among women after miscarriage in relation with age showed that 17 out of 93 cases ranges in 15-30 years of age; and 6 out of 51 were ranges in 31-49 years of age had depressive disorder without significant difference (Table I). Stratification with regard to educational status showed significant differences for educational status (Table I). Stratification with relation to family history showed borderline significance (Table I).

Annexure-l:

Symptoms needed to meet criteria for depressive episode in ICD-10

A

- Depressed mood

- Loss of interest

- Reduced energy and decreased activity

B

- Reduced concentration

- Reduced self-esteem and confidence

- Ideas of guilt and unworthiness

- Pessimistic thoughts

- Ideas of self-harm

- Disturbed sleep

- Diminished Appetite

Mild depression: At least 2 from $A$ and at least 2 from $B$

Moderate depression: At least 2 from $A$ and at least 3 from $B$

Severe depression: At least all from $A$ and at least 4 from $B$.

These symptoms will be assessed on ICD 10 depression inventory (approved by WHO) which is as follows: ICD 10 has categorised depression in three types. We include all these three categories in our study.

\section{The Major (ICD-10) Depression Inventory (MDI)}

The following questions ask about how patient have been feeling over the last two weeks.

Please put a tick in the box which is closest to how patient have been feeling.

\section{Bio-data:}

1. Patient name:

2. Age:

3. EDD:

4. Medical Record No.

5. Parity:

6: Previous miscarriage: Yes / No

\begin{tabular}{|c|c|c|c|c|c|c|c|}
\hline \multicolumn{2}{|c|}{ How much of the time... } & \multirow{2}{*}{$\begin{array}{c}\text { All of the time } \\
5\end{array}$} & \multirow{2}{*}{$\begin{array}{c}\text { Most of the time } \\
4\end{array}$} & \multirow{2}{*}{$\begin{array}{c}\begin{array}{c}\text { More than half } \\
\text { of the time }\end{array} \\
3\end{array}$} & \multirow{2}{*}{$\begin{array}{l}\text { Less than half } \\
\text { of the time } \\
2\end{array}$} & \multirow{2}{*}{$\begin{array}{c}\begin{array}{c}\text { Some of } \\
\text { the time }\end{array} \\
1\end{array}$} & \multirow{2}{*}{$\begin{array}{c}\text { At no time } \\
0\end{array}$} \\
\hline 1. & Have you felt in low spirits or sad? & & & & & & \\
\hline 2. & Have you lost interest in your daily activities? & 5 & 4 & 3 & 2 & 1 & 0 \\
\hline 3. & Have you felt lacking in energy and strength? & 5 & 4 & 3 & 2 & 1 & 0 \\
\hline 4. & Have you felt less self-confident? & 5 & 4 & 3 & 2 & 1 & 0 \\
\hline 5. & Have you had a bad conscience or feelings of guilt? & 5 & 4 & 3 & 2 & 1 & 0 \\
\hline 6. & Have you felt that life wasn't worth living? & 5 & 4 & 3 & 2 & 1 & 0 \\
\hline
\end{tabular}

7. Have you had difficulty in concentrating,

e.g. when reading the newspaper or watching television?

$8(a)$. Have you felt very restless?

8(b). Have you felt subdued?

9. Have you had trouble sleeping at night?

10(a). Have you suffered from reduced appetite?

10(b). Have you suffered from increased appetite?

Score interpretation:

For items 8 and 10 only the highest scored sub-item ( $a$ or $b$ ) is used.

Diagnosis of depression:

A score of 4 or 5 points in at least

2 of the first 3 items +2 of the first 7 items $=$ Mild depression ICD-10

2 of the first 3 items +4 of the first 7 items $=$ Moderate depression ICD-10

All of the first 3 items +5 of the first 7 items $=$ Severe depression ICD-10

1 of the first 2 items +5 of all 9 items = Major depression DSM-IV

Whether the patient falls in mild, moderate or severe category, they all will be the part of study. According to scores patient is depressive : Yes / No 


\section{DISCUSSION}

Abortion can result into high danger of having mental issues including depressive disorder, alcohol abuse or marijuana abuse, anxiety disorder and suicidal behaviour. The exact risk of depression associated with spontaneous-abortion in Asian population has not been established clearly. Only a few studies have showed the relation between grief and depression post-miscarriage.

As not much local data is available, however, the authors planned to highlight the frequency of depression in women after miscarriages and the results of study may be a guideline for gynecologists, obstetricians and medical physicians to emphasize on detection of depression after miscarriages in women and referral to mental healthcare / psychiatrist, so that the early diagnosis and treatment may be ensured, which will help in creating awareness and timely intervention.

These results were compared with a longitudinal cohort study, which was conducted in Hong Kong to determine the risk factors in 161 Chinese women post-miscarriage depressive disorder which was $10 \%$, anxiety disorder was $1.2 \%$, two subjects each had obsessive compulsive disorder and PTSD (0.6\%). Younger age, history of infertility and past history of depression are the vital risk factors after abortion. 9 The findings of our study are comparable with the study where mean 29.17 years, at a frequency of $15.33 \%$.

A recent study by Lock investigated the depressive disorder following spontaneous abortion. ${ }^{10}$ They recorded the mean age 30.39 years $(S D=6.38)$, which is similar to our findings. The prevalence of depressive disorder in spontaneous-abortion was $18.6 \%$, which is also comparable to this study.

Post-1990 revaluation of literature reporting decreased levels of depressive disorder is around 20\%.11-14 But in Western population, the percentage of depression after spontaneous-abortion was $40-50 \%$. However, the range of depression after spontaneous-abortion in Asian women may be less. The possible factors can be better societal support and a healthy relationship with the partner. Chinese women have the prevalence of depressive disorder six weeks after abortion as $12 \%$, while another report found prevalence of $10 \%$ after spontaneous-abortion (three months). ${ }^{15,16}$

In general population, social and cultural factors are influencing the prevalence of depression. A communitybased, cross-sectional analytical study was carried out in the urban areas of Colombo. The subjects were young elders from 60 to 74 years, who permanently resided in the area. The prevalence of depression was $13.9 \%$ (mild depression: $11.2 \%$; moderate to severe depression: $2.7 \%)$. Among the socio-demographic predictors, female gender of 60-64 years, being unmarried, separated, divorced, or widowed, income $\leq 20,000$ Sri Lankan rupees, were significantly associated with clinically significant depression. ${ }^{17}$

A study conducted in two different hospitals of Lahore proposed that social and spiritual support, religiosity and number of miscarriages will likely to predict distress in miscarrying women. Significant predictors of distress were found to be the increased number of miscarriages and social support. 18

The results of our study may permit healthcare workers to take proper interventions like guiding the family unit to provide emotional support to the women after miscarriage. Thus it will enhance the psychological and mental well-being of women or decreasing the effects of miscarriage on their relationships or work thus improving the overall quality of life.

\section{CONCLUSION}

The frequency of depressive disorder is not very high among women after miscarriage; however, by taking appropriate interventions like guiding the family unit to provide emotional support to the women after miscarriage may increase the psychological well-being of the women or decrease the harmful effects of miscarriage.

\section{ETHICAL APPROVAL:}

Ethical approval taken from Board of Review Services Hospital, Lahore.

\section{PATIENTS' CONSENT:}

Written informed consent was taken from every participant.

\section{CONFLICT OF INTEREST:}

Authors declared no conflict of interest.

\section{AUTHORS' CONTRIBUTION:}

AM: Major contributions to the conception or design of the work; or the acquisition, analysis, or interpretation of data for the work; and final approval of the version to be published.

AS:Drafting the work or revising it critically for important intellectual content; and final approval of the version to be published;

IU:Substantial contributions to the conception or design of the work.

SB: Primary concept and methodology defined plus data collection.

$A B$ : Writing work plus data search and assembling of the manuscripts.

FB: Provided data and SPSS usage and applying it.

NSK: Supervised the whole study and guided on every step.

\section{REFERENCES}

1. Francisco MdeF, Mattar R, Bortoletti FF, Nakamura MU. Sexuality and depression among pregnant women with recurrent spontaneous abortion. Rev Bras Ginecol Obstet $2014 ;$ 36:152-6. 
2. Rouse CE, Eckert LO, Babarinsa I, Fay E, Gupta M, Harrison MS, et al. Spontaneous abortion and ectopic pregnancy: Case definition \& guidelines for data collection, analysis, and presentation of maternal immunization safety data. Vaccine 2017; 35:6563.

3. Finer LB, Zolna MR. Declines in unintended pregnancy in the United States, 2008-2011. N Engl J Med 2016; 374:843-52.

4. Endler M. Characterizing retained placenta: Epidemiology and pathophysiology of a critical obstetric disorder. Thesis for doctoral degree. 2006.

5. Rich Daly depression risk after miscarriage often overlooked. [Access on 10 October 2014]. http://dx.doi.org/ 10.1176/pn.43. $12.0015 a$

6. Farren J, Jalmbrant M, Ameye L, Joash K, Mitchell-Jones N, Tapp S, et al. Post-traumatic stress, anxiety and depression following miscarriage or ectopic pregnancy: A prospective cohort study. BMJ Open 2016;6:e011864.

7. Meltzer-Brody S, Maegbaek ML, Medland SE, Miller WC, Sullivan P, Munk-Olsen T. Obstetrical, pregnancy and socioeconomic predictors for new-onset severe postpartum psychiatric disorders in primiparous women. Psychol Med 2017; 47:1427-41.

8. Kingsbury DM. The role of social networks in providing social support to resettled female refugees during their pregnancy in the United States (Doctoral dissertation, Kent State University).

9. Sham Ak, Yiu Mg, Ho Wy. Psychiatric morbidity following miscarriage in Hong Kong. Gen Hosp Psychiatry J Genhosppsych 2010; 32:284-93.
10. Lok IH, Yip AS, Lee DT. A 1-year longitudinal study of psychological morbidity after miscarriage. Fertil Steril 2010; 93:1966-75.

11. Kulathilaka S, Hanwella R, Varuni A. Depressive disorder and grief following spontaneous abortion. BMC Psychiatry 2016; 16:100.

12. Auerbach RP, Mortier P, Bruffaerts R, Alonso J, Benjet C, Cuijpers $\mathrm{P}$, et al. WHO World Mental Health Surveys International College Student Project: Prevalence and distribution of mental disorders. J Psychol Med 2018t; 127:623.

13. Srivastava S, Goyal A. Emotional support and psychological care of recurrent pregnancy loss couple. In Recurrent Pregnancy Loss 2018 (pp. 255-267). Springer, Singapore.

14. Mutiso SK, Murage A, Mukaindo AM. Prevalence of positive depression screen among post miscarriage women-A cross sectional study. BMC Psychiatry 2018; 18:32.

15. Hunter A, Tussis L, MacBeth A. The presence of anxiety, depression and stress in women and their partners during pregnancies following perinatal loss: A meta-analysis. J Affect Disord 2017; 223:153-64.

16. Zhu CS, Tan TC, Chen HY, Malhotra R, Allen JC, Østbye T. Threatened miscarriage and depressive and anxiety symptoms among women and partners in early pregnancy. $J$ Affect Disord 2018; 237:1-9.

17. Rajapakshe OB, Sivayogan S, Kulatunga PM. Prevalence and correlates of depression among older urban community dwelling adults in Sri Lanka. Psychogeriatrics 2019; 19:202-11.

18. Arshad T, Hafeez N. Religiosity social support and distress in maiscarriage. Pak J Prof Psychol 2016; 7:1. 